\title{
Atrioventricular, ventriculoarterial discordance and Ebsteinoid malformation of the tricuspid valve with severe regurgitation resulting in aortic atresia in a neonate
}

\author{
Balaganesh Karmegaraj ${ }^{1}$, Syed Ibrahim ${ }^{2}$, Mohamed Razeen Syed ${ }^{2}$, Ayesha Naseeha ${ }^{3}$, and \\ Balaji Srimurugan ${ }^{1}$ \\ ${ }^{1}$ Amrita Institute of Medical Sciences and Research Centre \\ ${ }^{2}$ Royal hospital \\ ${ }^{3}$ A.R. hospital
}

September 11, 2020

\begin{abstract}
Aortic atresia is uncommonly associated with atrioventricular and ventriculoarterial discordance.(1) Presence of severe regurgitation of Ebsteinoid malformation of the tricuspid valve in this subset results in reduced aortic blood flow in-utero. The hemodynamic explanation of this anomaly was reported by Celermajer and colleagues.(2) We report here a term neonate with this anomaly detect antenatally.
\end{abstract}

A 3000g term male neonate was referred for cardiac evaluation immediately after birth since fetal echocardiography detected congenitally corrected transposition of great arteries and Ebsteinoid malformation of the tricuspid valve at 27 weeks gestation. The baby had microcephaly and the cardiac examination a single second heart sound and mild destruction (Spo2 92\%). Bedside echocardiography showed atrioventricular discordance and atrialized right ventricle(RV) with Ebsteinoid malformation of the tricuspid valve(TV) on the four chamber view; There was severe regurgitation of the TV; [Figure 1A\&B] The ventricular septum was intact; There was ventriculoarterial discordance and no antegrade flow from RV to aorta;[Figure 1C] Parasternal long axis view showed aortic valve atresia with hypoplastic ascending aorta. [Figure 1D]Ductal arch view showed a huge patent ductus arteriosus(PDA) shunting entirely right to left from main pulmonary artery to descending aorta and the blood supply to aortic arch was entirely retrograde from the PDA. [Figure 1E] : High parasternal short axis view showing left and anterior position of aorta in relation to MPA (L posed aorta).[Figure 1F] Treatment options like primary heart transplantation or palliative surgery like Norwood operation with atrial septectomy were discussed with the parents. Considering the guarded long term outcome the parents opted for comfort care.

Hemodynamics of the described lesion: (2)

The combination of morphologic systemic right ventricle and severe systemic (Ebsteinoid malformation of Tricuspid valve) atrioventricular valve regurgitation resulting in compromised systemic forward flow in to the ascending aorta resulting in systemic outflow (aorta) atresia. The schematic cartoon demonstrating the hemodynamic explanation of the described lesion is shown in figure 2 . 

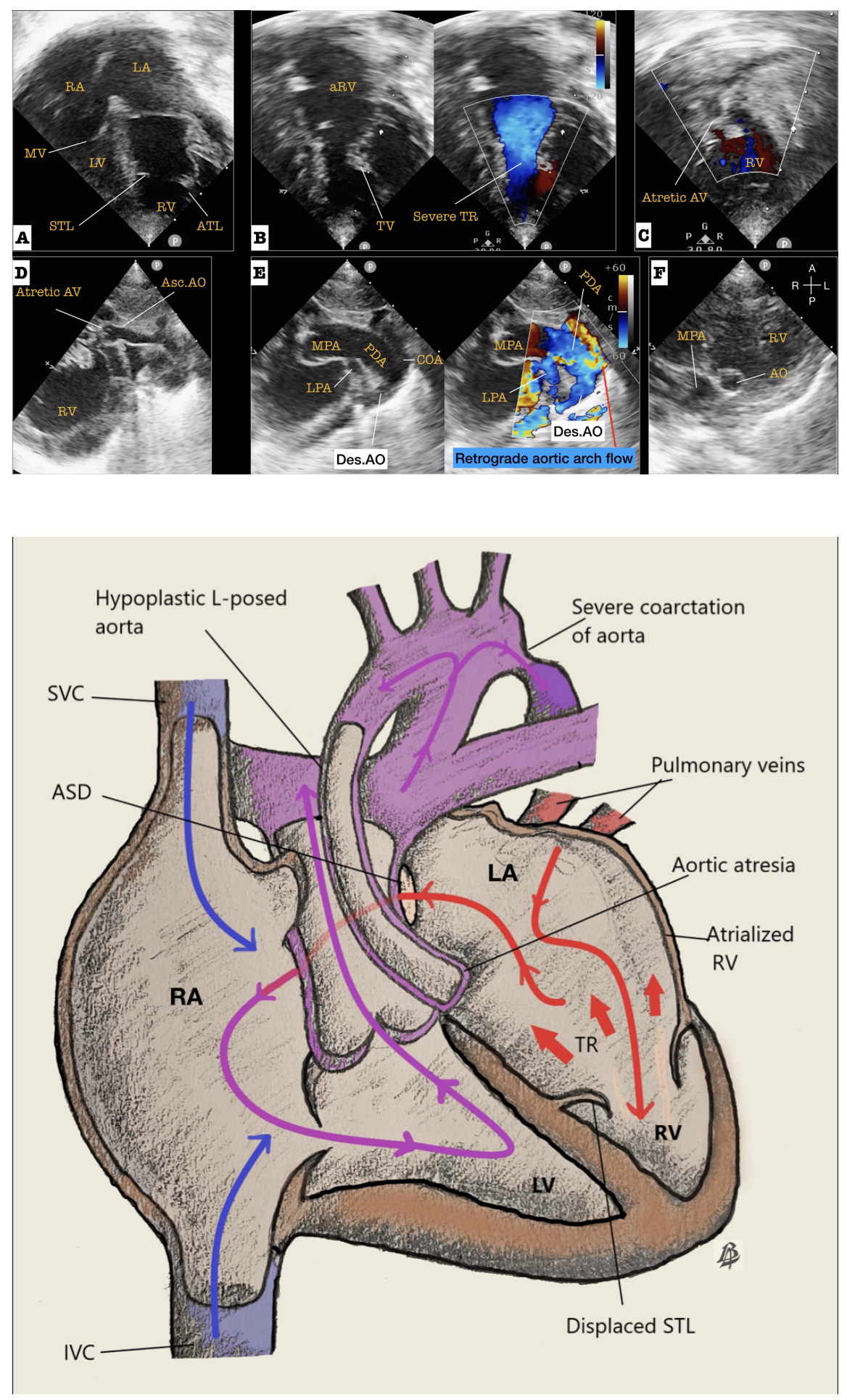